\title{
The Temporal Drift due to Polarization Noise in a Photonic Phase Reference Distribution System
}

\author{
P. Shen, N. J. Gomes, Senior Member, IEEE, W. P. Shillue, S. Albanna
}

\begin{abstract}
The temporal drift due to the polarization noise in an ultra-low temporal drift photonic phase reference distribution system is analyzed. The temporal drift is shown to be associated with the polarization changes, the differential group delay of the subsequent parts, and, the state of polarization dispersion of the photonic local oscillator signal. Such temporal drift cannot be corrected by Line Length Correction schemes. As an example, polarization changes from a fiber stretcher are presented, discussed, and identified as one of the major contributors to the system temporal drift. A numerical model is developed. The magnitude and statistical distribution (based on random coupling) of the temporal drift excited by the polarization change of the fiber stretcher are investigated. Experimental work demonstrates that reduction in the polarization change successfully reduces the temporal drift, as predicted by the theory and simulation.
\end{abstract}

Index Terms - phased array, polarization, phase jitter, frequency synthesizers, optical fiber, polarization mode dispersion, photonic local oscillator, phase reference system, temporal drift

\section{INTRODUCTION}

$\mathrm{O}$ PTICAL fiber has advantages of flexibility, broad bandwidth and ultra-low transmission loss. This makes it very attractive for long-span centralized clock signal distribution systems. With a transmission delay temperature coefficient of 8 to $10 \mathrm{ppm}$ per Celsius, typical telecom standard single mode fiber is generally regarded as a stable transmission medium [1-3]. When environmentally isolated, the stability is considerably greater than air paths, and no worse than that of coaxial cable. Hence, low loss fiber networks have been widely used or proposed for transporting frequency standards and timing signals $[4,5]$.

In high-end applications, a high quality continuous wave (CW) signal is often generated at a central site and then delivered to different remote sites through an optical fiber distribution network to maintain the phase synchronization of the different sites over relatively long time intervals. The centralized structure not only reduces cost, but also maintains the high degree of synchronization of the remote units, avoiding the relative drift between different signal sources. The

Manuscript received January 30, 2008. This work was supported by the ESO and ALMA project.

P. Shen is with the Electronics Department, University of Kent, Canterbury, Kent, UK. (e-mail: p.shen@kent.ac.uk).

N. J. Gomes is with the Electronics Department, University of Kent, Canterbury, Kent, CT2 7NT, UK.

W. P. Shillue and S. Albanna are with the National Radio Astronomy Observatory, Charlottesville, VA and Socorro, NM, respectively use of a low loss fiber network allows the signal to be distributed at great distance. Such a system is called a photonic phase reference distribution system. Ultra-low end-to-end temporal drift requirements on the phase reference distribution system present a technical challenge, yet are important in applications that require a high degree of coherence across a wide area. Such applications can be found in radio astronomy observation, particle accelerators, phased array radar, and frequency and clock signal distribution systems.

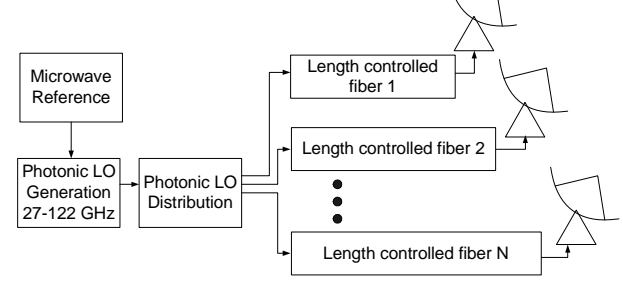

Fig. 1 Block diagram of the photonic phase reference distribution system in a radio astronomy project- Atacama Large Millimeter Array

As phase references, the distributed local oscillator (LO) signals must have very high temporal stability over a defined time scale, especially for millimeter- and submillimeter-wave applications. For instance, the Photonic Local Oscillator (PLO) distribution network in the Atacama Large Millimeter Array (ALMA) project [6] connects 66 antennas operating up to 950 $\mathrm{GHz}$ with PLO signals up to $122 \mathrm{GHz}$ used as phase references. This photonic millimeter wave LO reference signal has to be delivered over up to $16-\mathrm{km}$ of single mode fiber to the remote antennas, as illustrated in Fig. 1. The PLO signal is generated by heterodyning two highly coherent narrow linewidth lasers (master laser and slave laser) which are phase locked together, as shown in Fig. 2. The LO phase drift requirement is for a temporal stability of better than 12 fs over any period of 300 seconds. Obviously, the distribution paths are exposed to environmental perturbations on the fiber, such as vibration or temperature variation, as well as the movement of the fiber wrap that feeds into the antenna dish. A Line Length Correction (LLC) scheme [7,8] must be employed to compensate the group-delay variations caused by these effects.

This paper will attempt to describe the special requirements for photonic phase reference distribution systems which provide the highest stability. Apart from conventional effective length control of the distribution path, the polarization change excited drift becomes an important contributor to the overall temporal drift in these systems. Although Polarization Mode Dispersion (PMD) has been greatly explored for digital 
communications, its effect on analogue signal distribution has not been studied in detail. This paper provides the first detailed guidelines for the design of analog links in terms of polarization related issues. It is focused on how polarization change excited temporal drift affects system performance and on how it can be controlled and reduced in analog LO transmission systems. As an example the temporal drift associated with the polarization change in a fiber stretcher is addressed. By reducing the polarization change in the fiber stretcher, the system shows much reduced temporal drift. Both simulation and experiment results are presented.

\section{BACKGROUND}

\section{A. Fiber Link Stability}

While the phase jitter (high frequency noise) of the delivered LO signal is usually dominated by jitter from the PLO generator, the phase drift (low frequency noise) includes contributions both from the LO generator and from the distribution system. In a typical photonic phase reference distribution system where the temporal drift is important, the delivery fibers must be well insulated from environmental acoustic noise and temperature changes. However, long distribution networks can still accumulate a significant temporal drift in the signal over longer time scales across the network. For instance, for ALMA the uncorrected temporal drift is expected to be dominated by the contribution from above-ground sections of the fiber on the antennas, which could be as high as $0.5 \mathrm{~mm}$ per hour, and converts to $1.7 \mathrm{ps}$ per hour.

\section{B. Line Length Correction Scheme}

A high-precision LLC scheme may depend on successfully monitoring the round-trip phase of a reference lightwave passing through the fiber link and applying a correction to the transmission path through a feedback loop. A fiber stretcher is often used for the compensation of transmission delay as it provides a moderate tuning range and a speed up to $\mathrm{kHz}$ level. The detection of the lighwave phase error is carried out by mixing the reflected lightwave with the local reference light, as shown in Fig 2. The interference signal is detected by a photodetector and this carries the information of the round-trip delay. By stabilizing the interference pattern through length correction with a fiber stretcher, the round-trip delay can be locked. Frequency shifting of the round-trip lightwave by a low frequency allows the detection of the phase of a RF signal instead of the amplitude of the interference signal, thus avoiding problems such as the DC drift, optical power fluctuations, and spurious reflections. Due to the long loop delay and large phase excursions in the loop, techniques to improve the locking range and to achieve stable locking have been developed [9].

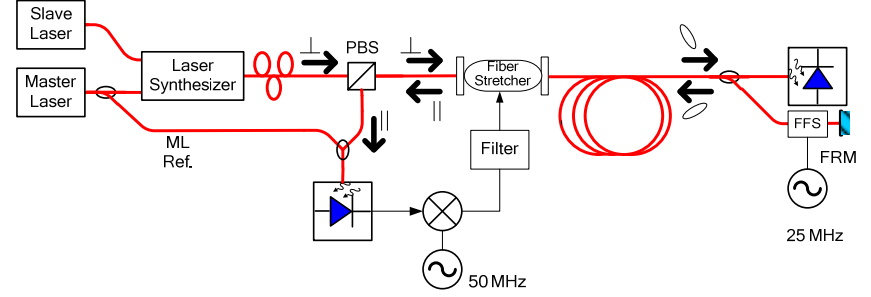

Fig. 2. Illustration of the LLC system [8]

The un-modulated master laser and slave laser are phase locked together. FFS: Fiber Frequency Shifter, FRM: Faraday Rotator Mirror, PBS: Polarization Beam Splitter

As the single mode fiber and components have nonzero birefringence, the polarization exiting the fiber link is not predictable, and subject to change in time, either due to perturbations in the fiber or due to the PMD evolution in time $[10,11]$. The sensitivity of the lightwave phase error detection depends on the heterodyning of the reference and reflected lightwaves, and therefore can be degraded by the drift of the polarization of the returned light. The extreme condition is when the two lightwaves are in orthogonal modes, and there is no detectable error signal at all. To overcome this problem, a Faraday Rotator Mirror (FRM) is included in the system. This polarization retracing configuration $[12,13]$ allows an unfaded reflected lightwave with stable known polarization (orthogonal to the launching polarization) to assist the error detection by maintaining the heterodyne efficiency. By using a stabilized narrow linewidth laser, FRM and fiber stretchers, the round-trip propagation time can be controlled to a fraction of the reference optical wavelength, i.e. sub- micrometer error in the effective length control, providing excellent temporal stability down to sub fs level.

\section{Polarization Mode Dispersion of the Optical System}

Single mode fiber is known to have PMD, thus supporting two degenerate modes, known as the Principal States of Polarization (PSPs). These two PSPs are orthogonal to each other when no Polarization Dependant Loss (PDL) is present in the system, and are sometimes referred to as the fast and slow modes. In summary, the PMD of the fiber has the following properties which affect the analogue link:

1) The value of the PMD is described by the Differential Group Delay (DGD) of the fiber at a particular wavelength, and the PSPs which represent the polarization orientation.

2) The PSP of the fiber link is not known unless measured, and is wavelength dependent.

3) The DGD is wavelength dependent. In general the lower the PMD of the link, the slower the DGD changes with wavelength since the PSP has a bandwidth related to the PMD of the link.

4) The magnitude of the DGD follows the Maxwellian distribution. For long installed fiber it has a mean value of

$$
\sigma_{\text {mean }-D G D}=\left(D_{p} \sqrt{L}\right)
$$

where the $D_{p}$ is the PMD coefficient of the fiber, and $\mathrm{L}$ is the fiber length. The PMD evolves in time due to environmental changes and aging of the fiber [14]. Both 
the magnitude of the DGD, and the orientation of the PSPs varies in time in a relatively unpredictable manner.

5) The lightwave passing through a system with PMD will suffer polarization dispersion in its output polarization between different spectral components. The term State of the Polarization Dispersion (SOPD) describes this polarization dispersion and its value is given by

$$
\begin{aligned}
& S O P D=\arccos \left(\frac{\overline{S_{\omega 1}} \cdot \overline{S_{\omega 2}}}{\left|\overline{S_{\omega 1}}\right| \cdot\left|\overline{S_{\omega 2}}\right|}\right) \\
& \leq D G D_{\lambda} \cdot \Delta \omega
\end{aligned}
$$

where $\omega$ is the optical angular frequency, $S$ is the Stokes Parameters of the polarization at different angular frequencies, $D G D_{\lambda}$ is the DGD at the local wavelength and $\Delta \omega$ is the frequency difference between the spectral components.

\section{Polarization RElated TEMPORAL DRIFT IN THE LLC}

Despite efforts that can stabilize the effective round-trip path length to within a fraction of a micrometer, the system can still be exposed to a number of additional temporal drift contributions associated with the polarization of the lightwave. In the presence of PMD, the transit time is no longer only related to the physical length and effective refractive index of the fiber link. The forward, backward and round-trip transit times will have dispersed values, subject to the polarization properties of the light and fiber. The immediate impact is that the round-trip delay is no longer twice the forward delay, and locking the round-trip delay does not produce any fixed time reference frame if the light is simply being reflected at the far end by an ordinary mirror.
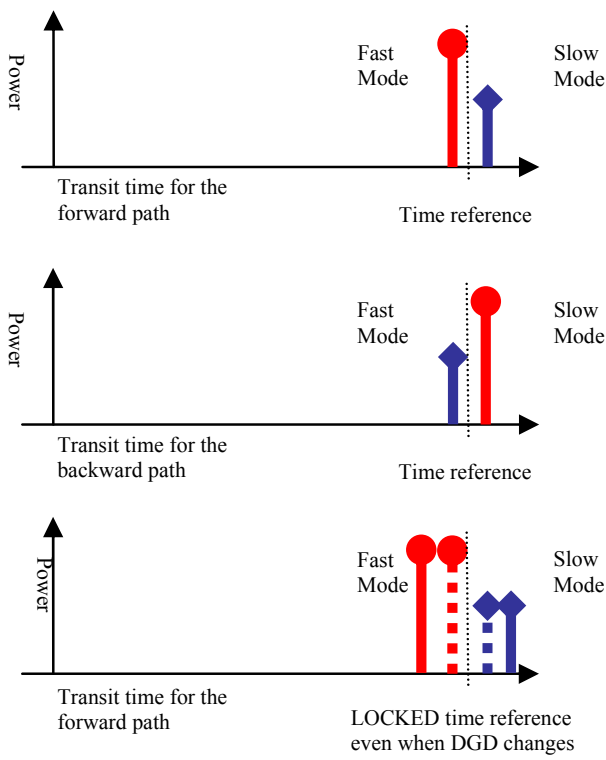

Fig. 3 Illustration of the forward transit time and the backward transit time in the LLC scheme with polarization retracing. The fast mode in the forward path turns into the slow mode in the backward path. The lower figure illustrates that when the DGD changes, the forward and backward transit times both change but their mean does not change if the round-trip delay is locked.
The polarization retracing system by using a FRM plays an important role in the LLC system in the presence of the DGD. If examined carefully, one can understand that the polarization retracing system in effect provides a round-trip time reference frame regardless of the PMD of the fiber link change. This is due to the fact that for any amount of PMD in the forward path, it is compensated in the return path and therefore the signal of the round-trip sees a zero overall link PMD. From the system point of view, any light launched in the fast mode of the fiber link will be returned in the slow mode and vice versa. This results in all polarization components seeing exactly the same propagation delay in the complete round-trip. When the round-trip is locked in this manner, the sum and hence the mean of the fast mode and the slow mode propagation times are locked.

When the PMD of the fiber or the polarization of the input light changes, the forward transit time will change even though the LLC maintains a perfect locking in the round-trip transit time of the fiber. For example, when the forward light is launched with all power in the slow mode, and the DGD magnitude increases, the light will arrive at the remote site later. This demonstrates how DGD change affects the distributed LO signal when the LLC is locked. The following equations show that the forward transit time is a function of the DGD of the fiber link.

$$
\begin{aligned}
& \tau_{D G D}=\frac{\tau_{s}-\tau_{f}}{2} \\
& \tau_{\text {round-trip }}=\tau_{f}+\tau_{s} \\
& \tau_{\text {effective }}=\frac{\tau_{f}+\tau_{s}}{2} \quad \text { LOCKED by LLC } \\
& \tau_{\text {forward }}=\tau_{s}=\frac{\tau_{\text {round-trip }}-\tau_{D G D}}{2} \\
& \tau_{\text {backward }}=\tau_{f}=\frac{\tau_{\text {round-trip }}+\tau_{D G D}}{2}
\end{aligned}
$$

Here $\tau$ represents the transit times, subscript $s, f$, round-trip and effective represent that of the fast mode, slow mode, round-trip and effective (or mean) values. The $\tau_{D G D}$ represents the DGD of the fiber link, given in the form of a time delay.

\section{Polarization Change EXCITEd TEMPoral DRIFT}

The optical receiving system contains a photodetector, a number of optical waveguides (such as fiber and monolithic waveguides guiding the light on the wafer), couplers, optical switches and an optical amplifier. These are not immune to polarization mode dispersion. When the input polarization to such an optical receiver varies, the detected signal is known to suffer amplitude and phase noise. This is known as the polarization noise, mostly studied as a form of the PMD effect on digital communications. This section is devoted to discussing polarization noise in analogue systems, which has not drawn much attention in the past.

\section{A. Magnitude of the Temporal Drift}

Since the coupling of the signal into the receiver's PSPs can change, the detected signal may suffer fading. When the 
polarization varies, the fading condition changes and results in the detected signal with varying amplitude and phase. The term State-of-Polarization-Change (SOPC) is employed here to describe the temporal evolution of the polarization states on the Poincare Sphere as the solid angle between the polarizations of time $t 1$ and $t 2$, respectively.

$$
S O P C=\arccos \left(\frac{\overline{S_{t 1}} \cdot \overline{S_{t 2}}}{\left|\overline{S_{t 1}}\right| \cdot\left|\overline{S_{t 2}}\right|}\right)
$$

For a dual wavelength heterodyne system exposed to a small SOPC, the temporal drift is within a range of [15]

$0 \leq \Delta \tau \leq \eta \cdot D G D_{R} \cdot \sin (S O P C)$

For a larger SOPC, the worst case can be estimated as

$$
0 \leq \Delta \tau \leq \begin{cases}\eta \cdot D G D_{R} \cdot 2 \sin (S O P C / 2) & \text { for } \mathrm{SOPC}<\pi \\ 2 \eta \cdot D G D_{R} & \text { for } \mathrm{SOPC} \geq \pi\end{cases}
$$

where $\eta$ is a weighting factor determined by the SOPD at the point of the excitation, and $D G D_{R}$ is the differential group delay of the subsequent part of the system at the central wavelength. The weighting factor is equal to 0.5 in the ideal case where the SOPD is zero, and increased slightly to 0.55 for the SOPD equal to 0.8 rads, which approximately corresponds to the case of a perfectly aligned $100 \mathrm{GHz}$ signal transmitted through 16 $\mathrm{km}$ of low PMD coefficient fiber (with a PMD coefficient of $0.1 \mathrm{ps} / \sqrt{\mathrm{km}}$ ) in the extreme PMD event. The further increase in the SOPD will cause the weighting factor to increase sharply as well as the degradation of the LO generation efficiency.

Equations $(4,5,6)$ have shown the range of the temporal drift excited by the polarization change. Among them the most important case is the system with a relatively small SOPD, since the SOPD will be limited by the requirement of maintaining the efficiency of the RF power generation anyway. Generally speaking, the worst scenario occurs when the two PSPs of the receiver are equally excited, and the transformation of the polarization states follows the great circle between the two PSPs. In the case that SOPD is present in the system, the worst scenario requires that the polarization states of each of the wavelengths is located on the same great circle with the mean polarization equally exciting the fast and slow PSPs.

\section{B. SOPC and DGD in the System}

The polarization instability in the system can originate from the signal generator, mechanical movement/stress of the fiber (such as re-orientation of the antenna, or adjustment of the optical delay lines in the LLC scheme) or due to the PMD evolution in any part of the system. The buried long fiber link contributes to polarization changes over very long time scales which can be ignored in general applications where only the coherence within a few minutes is important. The fiber stretcher and the antenna wraps are subject to changes well within observation time scales and therefore are more significant sources of the SOPC. The polarization drift of the PLO source must also be kept very stable to guarantee low-SOPC.

An un-optimized optical receiver that contains an optical pre-amplifier may have a DGD value of a few hundred fs. An optimized design with care taken to reduce the DGD value of the optical components should produce a receiver with an overall DGD value below $75 \mathrm{fs}$.

When the SOPC occurs at the central site, such as that from the PLO source or from the fiber stretcher, the buried fiber should be treated as part of the optical receiver. In this case the DGD of the buried fiber needs to be managed to limit the magnitude and speed of the polarization change. For this reason, the use of the high birefringence PM fiber in the distribution path is not a good idea. Single mode fiber with low PMD coefficient should be employed instead.

\section{SOPC OF THE FIBER STRETCHER}

The polarization changes in the fiber stretcher can excite the polarization noise associated temporal drift in the following fibers and receivers. Even with low PMD coefficient fiber, the PMD value in the long fiber link may still exceed the PMD value of the optical receiver. If the PMD value of the fiber link is dominant, the DGDR value is limited by the physical fiber length. Hence the tight temporal drift budget requires a low SOPC at the input to the fiber link, i.e. at the output of the fiber stretcher. In this section, the SOPC in the fiber stretcher is studied.

\section{A. SOPC Measurement Setup}

Fig. 4 shows the experimental setup used to characterize the SOPC of the fiber stretcher. The particular fiber stretcher under test has a full range of $3.0 \mathrm{~mm}$ when it is stretched to its maximum. It has an oval-like mandrel with an expansion gap at the straight part. Around $5 \mathrm{~m}$ of SM fiber is wound onto the mandrel. During the test the fiber stretcher is driven to give a stretching of $2.0 \mathrm{~mm}$ around its mid range. A polarimeter is used to measure output polarization while the fiber stretcher is being stretched. The SOPC can be then calculated by using (4).

As the SOPC is a function of the dynamic birefringence and the polarization launching condition, its value is expected to change for different input polarizations. A deterministic polarization controller (DPC) is employed to measure SOPC for each. The input polarization is determined by the DPC setting, and is pre-selected in a manner which results in the step-size between consecutive polarization points being no larger than $10^{\circ}$ on the surface of the Poincare Sphere. This results in 422 points altogether, and achieves good coverage of the input Poincare Sphere. It has been confirmed separately that the DPC output is polarization stable over hours, with an accuracy of $0.1^{\circ}$. To ensure the SOPC data measured are from the fiber stretcher only, all the fibers in the measurement are short SM fiber patchcords, are fixed to the optical bench, and are protected from direct air flow. 


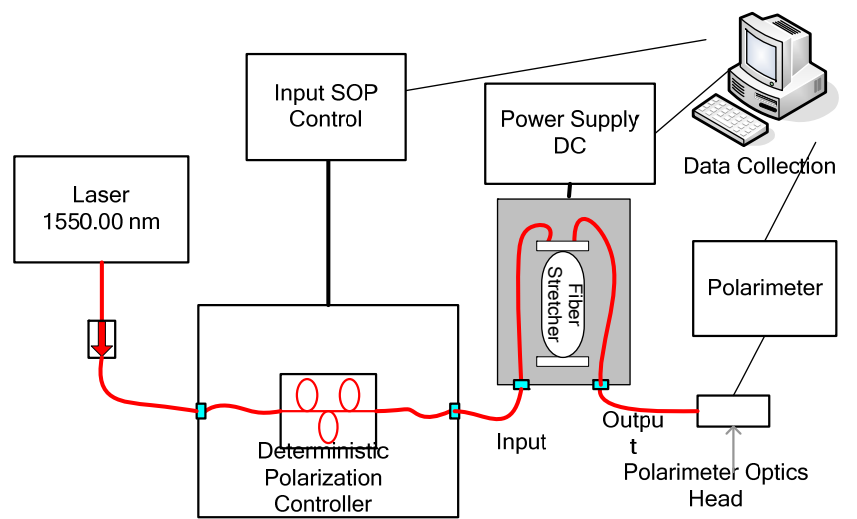

Fig. 4 Experimental setup to measure the SOPC at all possible input polarizations

\section{B. Measured SOPC of the Fiber Stretcher}

Fig. 5 depicts the calculated SOPC in a grayscale map, where the gray intensity indicates the magnitude of the SOPC for the corresponding input polarization. The coordinates of these points are plotted against the corresponding initial output SOP. When plotted on the Poincare Sphere, the fiber stretcher shows a quite regular SOPC pattern over the input polarizations, with two areas where the SOPC appears to be low. These two areas correspond to two special polarization states, which can be referred to as sweet-spots of the SOPC. Multiple tests have been carried out. The performance is repeatable when tested with the same fiber stretcher, and is similar when tested on other fiber stretchers with a similar structure, apart from the rotation of the polarization states due to the single mode fiber coupling in to and out of the fiber stretcher.

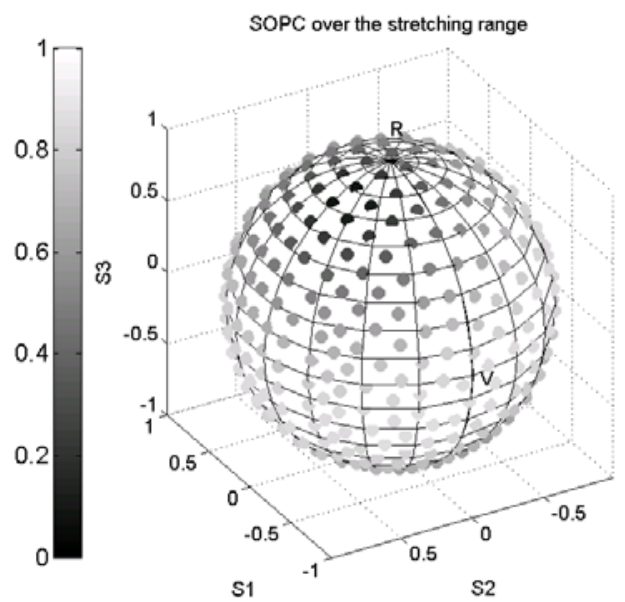

Fig. 5 The grayscale map of the SOPC of the fiber stretcher, corresponding to a $2 \mathrm{~mm}$ stretch, altogether 422 input polarizations.

The observed maximum SOPC is 1.0 radian for the worst case, over the $2 \mathrm{~mm}$ stretching range $( \pm 1.0 \mathrm{~mm}$ offset from the midrange). This occurs on only one occasion, at the very beginning of the test, and is believed due to the fiber stretcher's exposure to additional thermal shock due to the turn on of the devices, particularly the high voltage amplifiers inside the package.

Fig. 6 illustrates the histogram of the SOPC for the total 422 input polarizations. Fig. 7 compares the maximum and minimum SOPC case within the 422 input polarizations. The polarization change appears to be quite linear against stretching voltage. Generally speaking, with a random input polarization, it is more likely that an SOPC close to the maximum, 0.87 rads, will be observed. There are a few occurrences over the stretching range where the SOPC is very small, around 0.1 rads. This occurs when the input polarization is close to the sweet spots. This worst-case SOPC occurs at the locus of input polarizations that are midway between the two sweet-spots.

In the estimation of the impact that the fiber stretcher will make to the system, one needs to assume that the fiber stretcher has an expected SOPC that is equal to the maximum. With the subsequent fiber/receiver having an estimated PMD of around $300 \mathrm{fs}$, the use of (5) gives a worst case overall temporal drift of 63 fs per millimeter stretching. This value will occur when the polarization is orthogonal to the principle states of the following fiber and receiver, and may be somewhat less for other polarizations. Also, the rate of the drift depends on the rate of the fiber correction, which in turn depends on the site conditions, as that it may take 30 minutes or longer for this drift to take place. However, this still presents as a significant likelihood that the drift could reach 11 fs per 300 seconds.

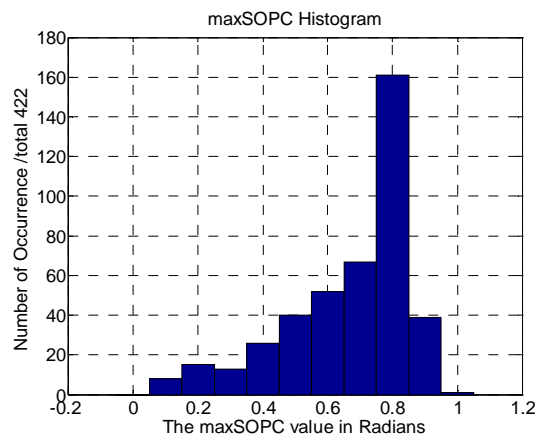

Fig. 6 The Histogram of the SOPC recorded over the $\pm 1 \mathrm{~mm}$ stretching range

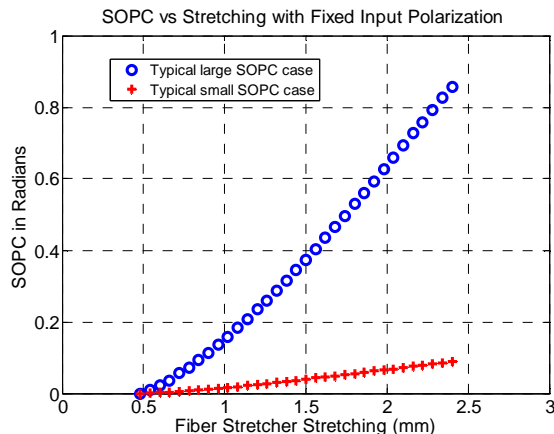

Fig. 7 Fiber Stretcher SOPC vs. stretching. A typical large SOPC case and a typical small SOPC case

\section{Simulation}

A numerical model based on VPI TransmissionMaker and Matlab has been developed to verify the temporal drift excited 
by the SOPC. Fig. 8 shows the block diagram of the model. For simplicity it is assumed that the two wavelengths are perfectly aligned at the PLO source, hence the SOPD at the input to the fiber stretcher is zero. The fiber stretcher is simulated by a piece of fiber with varying birefringence. The buried fiber is simulated by a coarse step method of scattering between random birefringent fiber sections. The mode coupling between the contiguous fiber components are set to be random unless specified. This reflects the situation that the PSPs of these components are normally unknown and can vary over along time or after reconnection. The receiver is simulated by a waveguide with a DGD prior to the ideal photo detector. Table III summarizes the simulation parameters.

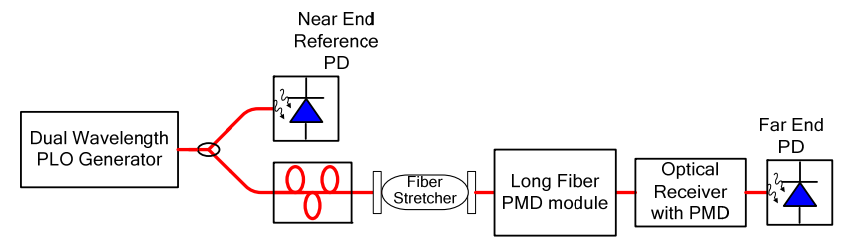

Fig. 8 Numerical model for the study of the fiber stretcher excited temporal drift

TABLE I

MAJOR PARAMETERS USED IN THE SIMULATION

\begin{tabular}{lc}
\hline \hline Items & Value \\
PLO Generator & \\
$\quad$ SOPD & 0 \\
Frequency & $100 \mathrm{GHz}$ \\
Fiber Stretcher & \\
$\quad$ Fiber length & $10 \mathrm{~m}$ \\
$\quad$ Dynamic birefringence & $1.65 \mathrm{fs}$ \\
Maximum SOPC & $2.0 \mathrm{rads}$ \\
Buried Fiber & \\
Length & $8 \mathrm{~km}$ \\
PMD coefficient & $0.1 \mathrm{ps} / \mathrm{sqrt}(\mathrm{km})$ \\
Dispersion & $17 \mathrm{ps} / \mathrm{km} / \mathrm{nm}$ \\
Receiver & \\
DGD & $75 \mathrm{fs}$ \\
\hline \hline
\end{tabular}

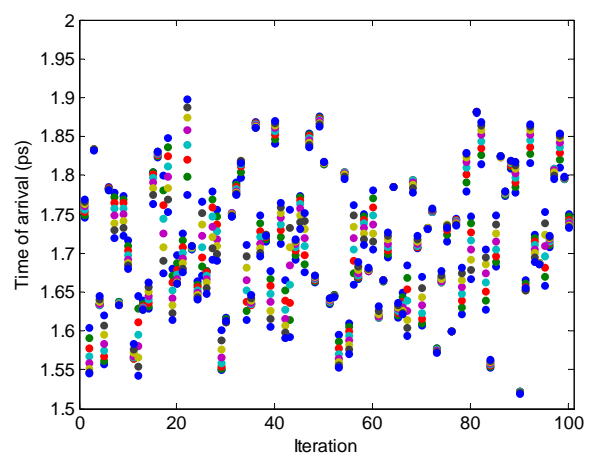

Fig. 9 The changes in the relative arrival time at the far end. Each group of data (for each iteration) corresponds to the fiber stretcher being stretched from minimum to maximum in a short period of time.

The simulation is taken by varying the birefringence of the fiber stretcher in each of the iterations, by an amount corresponding to stretching across its range. Within each iteration, the mode coupling between the components is set to be unchanged, representing the case that in a short time scale the optical path is unchanged. The difference between the iterations represents the variations of the links with the same parameters, due to the mode coupling variations.

Fig. 9 shows the recorded relative arrival time of the PLO signals for the first 100 iterations. Within each iteration, there are 8 points recorded, representing the time of arrival changes due to the temporal drift suffered by the PLO signal while the fiber stretcher is being stretched. The temporal drift is not a fixed value, due to the changes in the mode couplings and the variations within the buried fiber model from iteration to iteration.

The histogram of the temporal drift is presented in Fig 10a, which shows its statistical distribution. The inserted red line represents a limit for the maximum temporal drift due to the polarization change according to (5), by assuming the overall DGD of the receive section following the stretcher is:

$$
D G D_{R} \cdot=\sqrt{P M D^{2}{ }_{\text {fiber }}+D G D_{R c}^{2}}
$$

where the $D G D_{R c}$ is the averagely estimated DGD of the photo-receiver at the remote site, and the DGD of the fiber is equal to the PMD value of the fiber .

It is clear that the temporal drift stays within the range predicted for most of the cases. The instantaneous value of the DGD of the fiber/receiver can be larger than calculated value due to: a) the PMD statistics and b) potentially unfavorable mode coupling between the fiber and the receiver. Thus, there is a small probability that the temporal drift will exceed the maximum value estimated by (5) and (7).

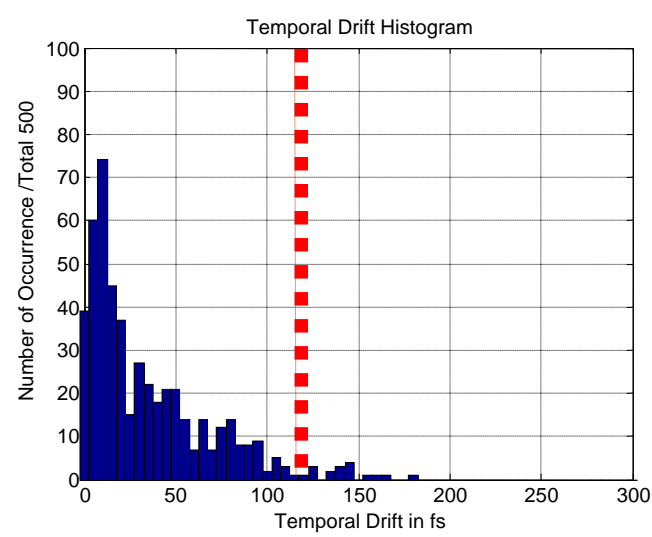

(a)

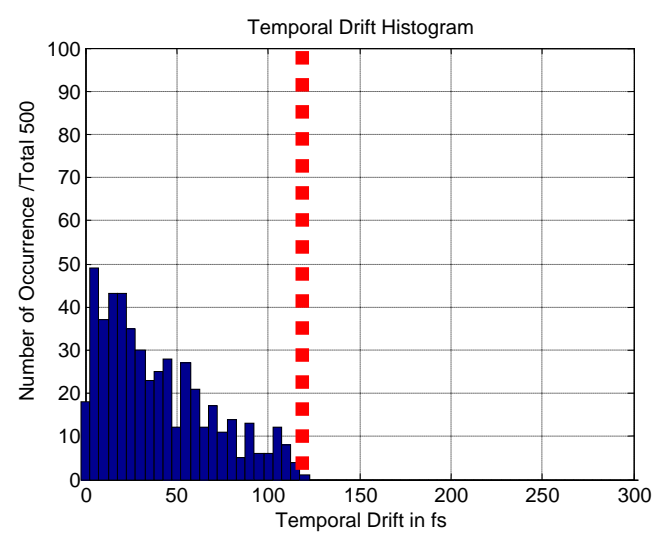


(b)

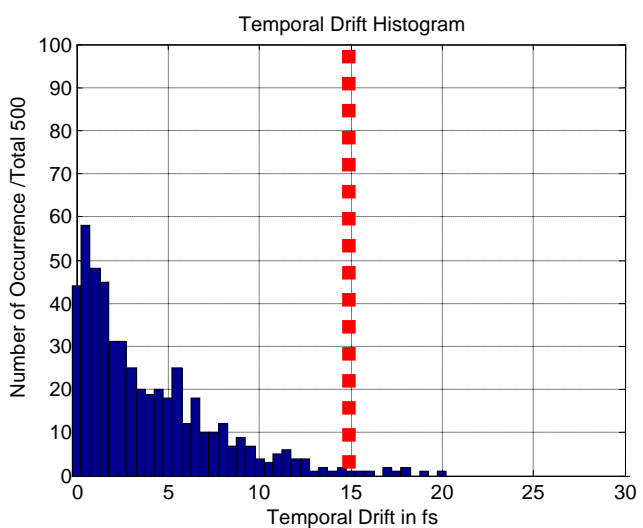

(c)

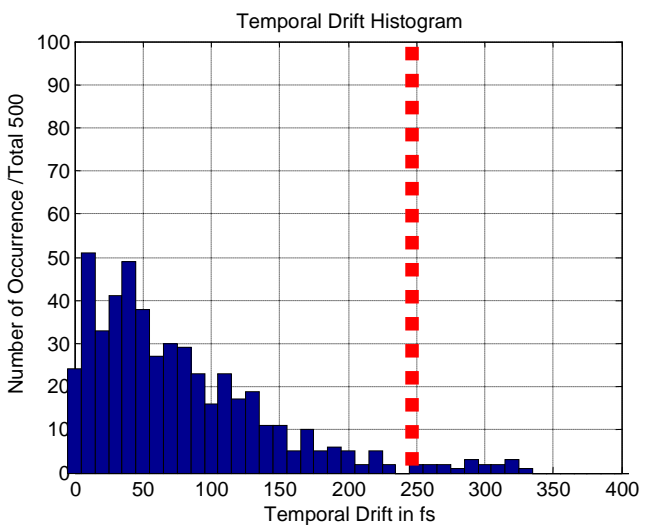

(d)

Fig. 10 Histogram of the temporal drift, a: maximum stretcher SOPC 0.9 rads with random mode coupling, b: maximum stretcher SOPC 0.9 rads with fixed DGD receiver of $293 \mathrm{fs}$. c: maximum stretcher SOPC 0.1 rads with random mode coupling d: maximum stretcher SOPC 2.0 rads with random mode coupling Inserted red lines are for the prediction based on (5) (6) and (7)

For comparison the simulation is run again with the DGD of the fiber and receiver set to be a fixed value. In this case the overall DGD of the receiving parts are set to be $293 \mathrm{fs}$, the estimated value according to (7). The histogram of the simulated temporal drift is then plotted in Fig.10.b. In this case no exception has been observed.

In order to reduce the polarization change excited temporal drift, one can choose to reduce the DGD or the SOPC, or both. There are technology limitations in further reducing the DGD of the buried fiber and the receiver. The SOPC of the fiber stretcher however can be improved by different methods such as improving the design of the fiber stretcher in its birefringence change, or by aligning the input polarization to one of its sweet spots. The latter will be reported in a separate paper. Here we present a case where the birefringence change in the fiber stretcher is reduced so that the maximum SOPC of the fiber stretcher is smaller.

Fig. 10.c shows the PLO temporal drift distribution for such a case by replacing the fiber stretcher model with one that has less birefringence changes. It generates a maximum SOPC of 0.1 rads, while keeping the remainder of the system parameters unchanged. It is obvious that the temporal drift in this case is about 10 times smaller, but their magnitude follows a similar distribution to that in Fig. 10. a. For comparison, a case with a SOPC of 2.0 rads is also presented in Fig. 10.d. Comparing Fig. a, c, d, the temporal drift reduction by reducing the SOPC is evident.

\section{EXPERIMENTAL RESULT}

An experiment is setup to verify the predictions. As shown in Fig. 11, the test consisted of two warm cartridge assemblies (WCAs) phase locked to photomixers at $81 \mathrm{GHz}$. The WCA is a solid-state millimeter-wave oscillator assembly which allows for an offset phase-lock so that the WCA output frequencies can be slightly $(31 \mathrm{MHz})$ offset from each other. The resultant phase difference is measured between one $14 \mathrm{~km}$ arm with an active LLC correction, and one short uncorrected fiber length. Two fiber stretchers are inserted into the LLC system, as shown in the inset of Fig. 11. The two fiber stretchers can excite SOPC of 2.0 and 0.1 rads, respectively. Tests were performed alternately with one stretcher activated and the other inactive. In this way, two datasets were acquired, one with a large SOPC and one with small SOPC.

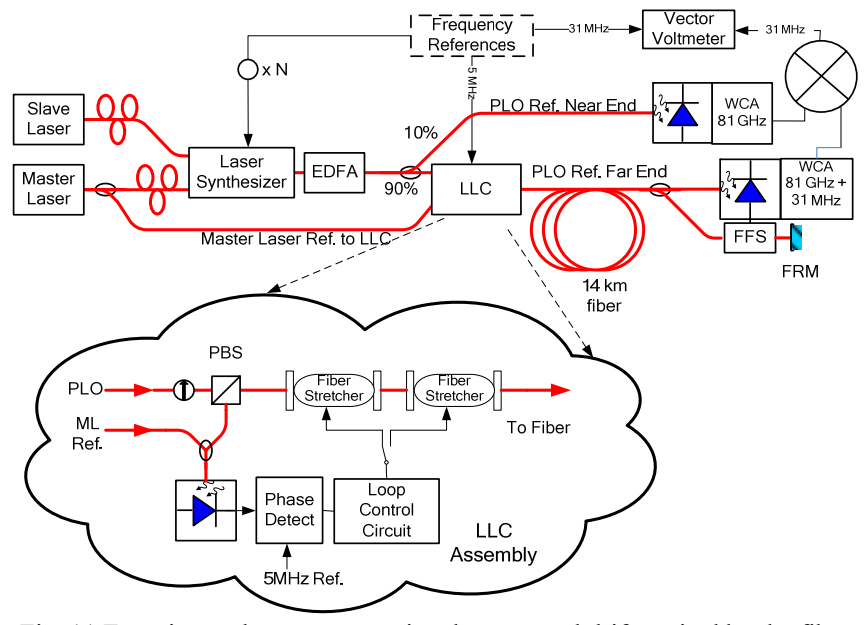

Fig. 11 Experimental setup to examine the temporal drift excited by the fiber stretcher SOPC

FFS: Fiber Frequency Shifter, EDFA: Erbium Doped Fiber Amplifier

Fig. 12 shows the first experiment in which the stretcher with large SOPC is activated. The $8 \mathrm{~km}$ spool of fiber is warming up, therefore creating a length change that forces the fiber stretcher to act. In Fig 12 the stretcher voltage is shown going through two cycles of its entire dynamic range. The SOPC and the residual phase of the system are shown to be clearly oscillating. (Note that phase jumps due to stretcher resets have been removed).

The 2.0 radian SOPC change causes a phase change of about $2 \mathrm{deg}$ at $81 \mathrm{GHz}$. The equivalent temporal drift (69 fsec) fits within the expected range shown in Fig. 10. d. There is also a slow drift variation of the residual phase which is thought to be due to uncorrected fiber. The portion of the curve where it appears that the fiber stretcher is saturated actually corresponds to the period during which the high SOPC is de-activated and the low-SOPC stretcher is activated instead. During this portion of the phase correction the phase oscillations are 
decreased as expected.

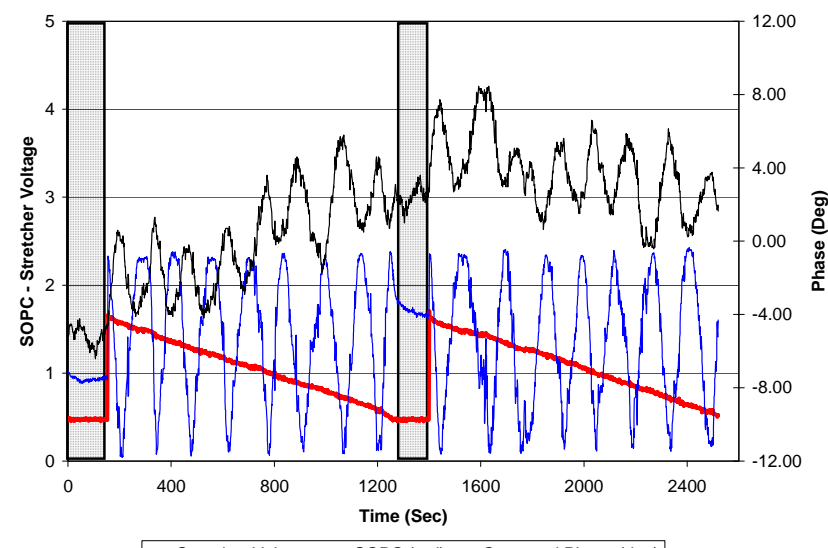

Fig. 12 Measured PLO phase and SOPC versus time for the fiber stretchers with large SOPC and small SOPC. The gray area shows the time intervals when the fiber stretcher with a small SOPC is active

This is shown more clearly in Fig. 13. In this case only the stretcher with low SOPC is used. The test fiber spool used in this case was $14 \mathrm{~km}$, but the test setup was otherwise the same. The figure clearly shows much lower SOPC than in Fig. 12, and also a much smaller residual phase oscillation. The phase oscillation is difficult to see because of the second order effect of residual phase due to the temperature change on uncorrected fiber.

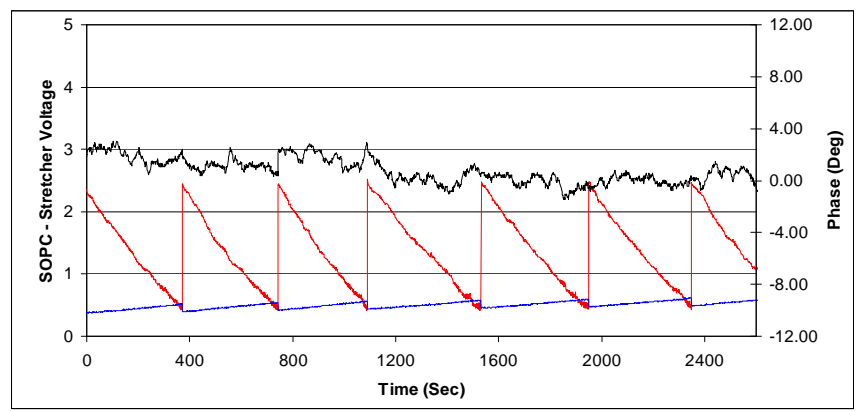

Fig. 13: Measured PLO Phase and SOPC versus time for the fiber stretcher with small SOPC

\section{CONCLUSION}

In this paper, the polarization associated noise on an ultra-low temporal drift phase reference system has been examined. Equations estimating the range of the temporal drift due to the polarization change have been presented which links the magnitude of the temporal drift to the polarization changes and the DGD in the system. This allows improvements to system design to be performed accordingly. The SOPC characteristics of a fiber stretcher have been studied. Both simulation and experiment confirm that reducing the SOPC in the fiber stretcher can reduce the temporal drift. Further work on reducing the SOPC in the fiber stretcher is being undertaken and the results will be published in a separate paper.

\section{References}

1. Lutes, G., and W. Diener, "Thermal coefficient of delay for various coaxial and fibre-optic Cable," TDA Progress Report, 42-99, Nov,1989, pp. 43-59, 1989

2. Roger Norrod, "Measurement of optical fiber temperature coefficient of Delay," National Radio Astronomy Observatory, Electronics Division Technical notes \#168, 1993

3. J. Ye, J. Peng, R. J. Jones, K. W. Holman, J. L. Hall, D. J. Jones, S. A. Diddams, J. Kitching, S. Bize, J. C. Bergquist, L. W. Hollberg, L. Robertsson, and L. Ma, "Delivery of high stability optical and microwave frequency standards over an optical fiber network," J. Opt. Soc. Am. B, vol. 20 pp. 1459-1467, 2003

4. T. P. Celano, S. R. Stein, G. A. Gifford, B. A. Mesander, and B. J. Ramsey, "Subpicosecond active timing control over fiber optic cable,", Proceedings of the 2002 IEEE International Frequency Control Symposium (IEEE, New York, 2002), pp. 510-516, 2002

5. H. Ogawa, D. Polifko, and S. Bamba, "Millimeter-wave fiber optics systems for personal radio communications," IEEE Trans. Microw. Theory Tech., vol., 40pp. 2285-2293, 1992

6. A. Wootten, "The Atacama Large Millimeter Array (ALMA)," in Proc. SPIE, vol. 4837, 2003, pp. 110-118, 2003

7. J. Cliche and B. Shillue, "Precision Timing Control for Radioastronomy, Maintaining Femtosecond Synchronization in the Atacama Large Millimeter Array", IEEE Control Systems Magazine, pp. 19-26, 2006

8. B. Shillue, S. AlBanna, and L. D'Addario, "Transmission of low phase noise, low phase drift millimeter-wavelength references by stabilized fiber distribution system", IEEE Intl. Topical Meeting on Microwave Photonics, MWP 2004, pp. 201-204, 2004

9. J. Levine, "Introduction to time and frequency metrology," Rev. Sci. Instrum. vol. 70, pp. 2567-2596, 1999

10. V. Ramaswamy, "Polarisation effects in short length, single mode fibres", Bell Systems Technical Journal, pp. 635-651, 1978

11. O. Karlsson, "Long-term measurement of PMD and polarization drift in installed fibres", J. Lightwave Technology, vol. 18, pp.941-951, 2000

12. N. C. Pistoni and M. Martinelli, "Polarization noise suppression in retracing optical fiber circuits", Optics Letter pp. 711, 1991

13. M. Martinelli, "A Universal Compensator for Polarization Changes Induced By Birefringence on a Retracing Beam," Optics Communication, vol. 72, pp. 341-344, 1989

14. M. Brodsky, "Polarization-Mode Dispersion of Installed Recent Vintage Fiber as a Parametric Function of Temperature", Photonics Technology Letters, vol. 16, pp. 209 -211, 2004

15. P. Shen, N. J. Gomes, P. A. Davies, and W. P. Shillue, "Polarization Mode Noise in Ultra-low Drift Phase Reference Distribution System over a Fiber Network," IEEE Intl. Topical Meeting on Microwave Photonics, MWP2005, pp. 297-300, 2005 